\title{
Effect of microtopographic structures of silk fibroin on endothelial cell behavior
}

\author{
JIN-YUN TAN ${ }^{1}$, JIAN-CHUAN WEN ${ }^{2}$, WEI-HAO SHI ${ }^{1}$, QING HE ${ }^{1}$, LEI ZHU ${ }^{1}$, \\ KUN LIANG $^{1}$, ZHENG-ZHONG SHAO ${ }^{2}$ and BO YU ${ }^{3}$ \\ ${ }^{1}$ Department of Surgery, Huashan Hospital of Fudan University, Shanghai 200040; \\ ${ }^{2}$ Laboratory of Advanced Materials of Fudan University, Shanghai 200438; \\ ${ }^{3}$ Department of Surgery, Pudong Hospital of Fudan University, Shanghai 201399, P.R. China
}

Received August 24, 2012; Accepted October 5, 2012

DOI: $10.3892 / \mathrm{mmr} .2012 .1127$

\begin{abstract}
Stent implantation has become the preferred revascularization treatment for occlusive blood vessel disease; however, there are occasionally complications resulting in re-narrowing of the treated artery. One approach to overcoming this problem is to establish a confluent monolayer of endothelial cells (ECs) on the stent, and a coating would facilitate the attachment of ECs. Silk fibroin was reported to be used as an ideal coating applied to stent for the culture of human ECs. The aim of the present study is to gain more insight into the influence of the internal microtopographical structure of silk fibroin on cell behavior, such as attachment and growth, and to further investigate its molecular mechanism using human umbilical vein ECs (HUVECs). Our results evaluated the effect of different microtopographical structures on cell behavior. In addition, we analyzed the cell cycle and investigated relevant molecules involved. The results indicated that the microtopographic structure of silk fibroin was associated with EC morphology, attachment and proliferation.
\end{abstract}

\section{Introduction}

A new era commenced when metallic stents were firstly introduced into clinical practice for the management of coronary and peripheral artery disease following transluminal angioplasty. This development brought about a significant reduction in restenosis compared to balloon angioplasty (1). Stent implantation has improved outcomes and become the preferred revascularization treatment for occlusive blood vessel disease. However, during surgical intervention, the unavoidable vascular trauma may initiate undesirable

Correspondence to: Professor Bo Yu, Department of Surgery, Pudong Hospital of Fudan University, 2800 Gongwei Road, Pudong New District, Shanghai 201399, P.R. China

E-mail: paul.yubo@gmail.com

Key words: silk fibroin, microtopographic structure, endothelial cell behavior, molecular mechanism responses such as the excessive proliferation of vascular smooth muscle cells (SMCs), extracellular matrix synthesis, thrombosis and a chronic inflammatory reaction, leading to complications resulting in the re-narrowing of the treated artery, known clinically as in-stent restenosis (ISR) (2). ISR is defined as diameter stenosis of $\geq 50 \%$ in the stented area of the vessel mainly due to excessive neointimal proliferation within the stented segment. This reduces the long-term efficacy of metal stent implantation to $20-40 \%$.

ISR represents an abnormal vascular response and repair to injury that results in excessive tissue growth. Angioscopic and pathological evidence suggests that endothelial cell (EC) dysfunction contributes to such development (3). A healthy endothelium sustains an antithrombotic milieu by secretion of various factors that exert antiaggregatory effects on platelets or have anticoagulatory or fibrinolytic properties (4). When in a confluent monolayer, ECs cease replication. Following stent implantation, the attendant endothelial denudation allows thrombus formation in response to exposure of highly thrombogenic substances from ruptured or erosive plaques, and in the meantime, the SMCs proliferate and migrate to the deendothelialized vessel surface, where they continue to proliferate and secrete extracellular matrix proteins, leading to neointimal tissue formation.

A possible future approach to overcome ISR is to promote and accelerate reendothelialization in the injured vessel, which is more natural and consequently safer. Among them, EC-capture stent, a type of stent used to attract ECs, would ultimately promote the elution of biologically active substances through a functioning endothelium monolayer, revealing correlation with a decrease in thrombosis and intimal thickening (5). Endothelium cell seeding techniques could be of high value for the establishment of a confluent monolayer on the stent in vitro. However, in vitro experiments showed that limited ECs were retained after stent expansion at the time of implantation and the subsequent pulsatile flow exposure $(6,7)$.

Prior studies have suggested that coating the stent with a matrix such as fibronectin or collagen would facilitate the attachment of ECs. These studies have also demonstrated that fibronectin-coated stents can be seeded in vitro with vascular ECs $(8,9)$ and that a number of the cells remain adherent to the stent following balloon expansion of the stent (10). However, 
these matrices are thrombogenic and could increase stent thrombogenicity. In addition, their poor mechanical property and limited ability to sustain stent expansion and deployment are also substantial challenges in clinical application $(10,11)$.

A number of other different compounds have been utilized as scaffold for the growth of ECs in the last decade. Among them, silk fibroin, the material that remains after the removal of sericin from silk, has demonstrated unique mechanical properties as well as excellent biocompatibility, controlled degradability and versatile processability in different material formats, and could be used to coat the stent in an attempt to increase attachment to ECs $(12,13)$. Silk has been used for centuries primarily as suture material. Its fibers are composed of two major types of protein: a) sericin, the antigenic gumlike protein surrounding the fibers; and b) fibroin, the core filaments of silk responsible for silk's elasticity (14). If sericin is removed, purified silk fibroin exhibits few immunological reactions, retaining the strength and many other desirable features of silk just as mentioned above.

Recent studies have revealed that physical modifications of biomaterials may influence cell reaction (15), and that nanostructured materials have been shown to support endothelial cellular attachment and growth (16). Thus, to validate silk fibroin's ability for coating the stent, it is our belief that a deeper understanding of cell-material interactions is essential for controlling endothelial cellular function by the physical parameters of the material. In the present study, we focused on employing a micromolding technique to produce silk fibroin with different microstructural features at micron scale, and at the same time, to evaluate the effect of these microtopographical features on cell behavior, such as attachment and growth, using human umbilical vein ECs (HUVECs). Furthermore, we analyzed the cell cycle and investigated relevant molecules involved in the cell growth and adhesive molecules such as integrin $\alpha 5 \beta 1$ and $\alpha 5 \beta 3$ for the study of the biological performance of cells on different patterned surfaces.

\section{Materials and methods}

Fabrication of silk fibroin. Silk fibroins with different microtopographical features were kindly supplied by the Laboratory of Advanced Materials, Fudan University. Polydimethylsiloxane (PDMS) molds were surface modified by carving various combinations of grooves and ridges into the surface using a computer controlled excimer laser beam through a metal photomask. The mask was not in close contact with the surface and the desired pattern was projected via the laser beam onto the area of interest. The silk fibroin solutions prepared earlier were pipetted on top of the pattern present on the PDMS mold. As the silk fibroin solution evaporated and transformed to membrane, the micropatterns of the PDMS mold were transferred to the membrane. In this way, 4 types of silk fibroin were obtained having microtopographical structures at micron scale, as well as one control, having a non-patterned structure.

Cell culture on the silk fibroin surfaces. HUVECs were isolated according to a previously published method (17). Cells were cultured in medium M199 (Sigma, St. Louis, MO, USA) supplemented with $20 \%$ fetal bovine serum (Thermo Scientific Hyclone, South Logan, UT, USA). Cells were seeded at a density of $1 \times 10^{4}$ cells $/ \mathrm{cm}^{2}$ in a 6 -well plate containing 4 types of silk fibroin with different microtopographic structures and one with a non-patterned structure. The plate was incubated at $37^{\circ} \mathrm{C}$ in $5 \%$ carbon dioxide. Cells were cultured for 3 days and were imaged at x160 magnification using an IX-71 microscope (Olympus, Tokyo, Japan) equipped with a high resolution digital camera.

Cell proliferation assay. Cell proliferation was evaluated during culture time $(24,48$ and $72 \mathrm{~h}$ ) to detect the effect of microtopographic structures on the proliferation of HUVECs by Sulforhodamine B (SRB). Cells $\left(5 \times 10^{3}\right.$ cells/well in $100 \mu 1$ medium) were seeded in 96-well plates containing the 4 types of silk fibroin with different microtopographic structures and one with a non-patterned structure. After each time point, $50 \mu \mathrm{l}$ of $30 \%$ trichloroacetic acid was added for $60 \mathrm{~min}$ at $4^{\circ} \mathrm{C}$. After washing and drying the plate, $100 \mu \mathrm{l}$ of $0.4 \%$ SRB was added for $30 \mathrm{~min}$. The plates were rinsed with $0.1 \%$ acetic acid and air-dried, after which $100 \mu \mathrm{l}$ of Tris base $(10 \mathrm{mmol} / \mathrm{l})$ was added, and the plates were agitated for $5 \mathrm{~min}$. The SRB value was measured at a wavelength of $490 \mathrm{~nm}$ by iMark ${ }^{\mathrm{TM}}$ Microplate Reader (Bio-Rad, Hercules, CA, USA). The experiment was performed in quintuplicate and repeated three times.

Real-time PCR analysis. HUVECs were seeded in $60 \mathrm{~mm}$ plates $\left(1 \times 10^{6} /\right.$ plate) containing the 4 types of silk fibroin with different microtopographic structures and one with a non-pattened structure. After incubation for $72 \mathrm{~h}$, cells were removed from plates with trypsin and then centrifuged. The total cellular RNA was extracted from the cell pellets using TRIzol (Invitrogen, Carlsbad, CA, USA) and then transcribed into cDNA with a RevertAid ${ }^{\mathrm{TM}}$ first strand cDNA synthesis kit (Fermentas, Vilnius, Lithuania) using $2 \mu \mathrm{g}$ total RNA and oligo(dT) primers. The quantitative PCR included $2 \mu \mathrm{l}$ cDNA and $10 \mu \mathrm{l}$ SYBR-Green Master mix (Takara, China) with a pair of primers. The reactions were monitored on the ABI PRISM 7500 sequence system (Applied Biosystems, Carlsbad, CA, USA). mRNA levels of VEGF, VCAM-1, Eselectin, $\alpha 5 \beta 3$ and E-cadherin were calculated using the equation $2^{-\Delta \Delta C t}$ and normalized to human GAPDH mRNA levels. The primer sequences for specific mRNA are shown in Table I.

Western blot analysis for adhesive molecules. HUVECs were seeded in 60-mm plates as described above. Following incubation for $72 \mathrm{~h}$, cells were removed from the plates with trypsin and then centrifuged. Cell pellets were resuspended in lysis buffer (1\% Triton X100, $50 \mathrm{mM}$ Hepes, $\mathrm{pH} 7.4,150 \mathrm{mM} \mathrm{NaCl}$, $1.5 \mathrm{mM} \mathrm{MgCl} 2,1 \mathrm{mM}$ EGTA, $100 \mathrm{mM} \mathrm{NaF}, 10 \mathrm{mM}$ NaPPi and $10 \%$ glycerol to which $1 \mathrm{mM}$ PMSF, $1 \mathrm{mM} \mathrm{Na} \mathrm{VO}_{4}$, and $1 \mathrm{X}$ protease inhibitor were added before use) to harvest cell lysates. Proteins from total cell lysates were separated using a 10-15\% SDS-PAGE gel and then transferred to PVDF membranes (Millipore, Billerica, MA, USA). The membranes were blocked, washed, and incubated with specific primary antibodies. The primary antibody incubation was followed by incubation with HRP-conjugated secondary antibodies. The bands were detected with an enhanced chemiluminescence assay (PerkinElmer, Waltham, MA, USA). The protein expression level of $\alpha 5 \beta 1$ and $\alpha 5 \beta 3$ was calculated using Quantity One software (Bio-Rad). 
Table I. Primer sequences for specific genes.

\begin{tabular}{ll}
\hline Gene & \multicolumn{1}{c}{ Primer sequences (5'-3') } \\
\hline VEGF & F: AATCATCACGAAGTGGTGAAG \\
& R: AATCTGCATGGTGATGTTGGA \\
VCAM-1 & F: TTAGATAATGGGAATCTACA \\
& R: TCAACATGACTGAGTCTCCAA \\
Eselectin & F: TGAGTGTGATGCTGTGACAAA \\
& R: TTCTGAGGCTGGCGGACGG \\
$\alpha 533$ & F: TTAAGAATGCTTACAATAA \\
& R: AACGACTGCTCCTGGATGCAC \\
E-cadherin & F: TTGCTCACATTTCCCAACTCC \\
& R: TTTCAATAATAAAGACACCAA \\
GAPDH & F: TTCACCACCATGGAGAAGGCTG \\
& R: TTCCACGATACCAAAGTTGTCA
\end{tabular}

$\mathrm{F}$, forward; $\mathrm{R}$, reverse.

Enzyme-linked immunosorbent assay (ELISA) test. HUVECs were seeded in $60-\mathrm{mm}$ plates as described above. Supernatants were harvested after $72 \mathrm{~h}$ and centrifuged at 2,000 x g to measure the concentration of protein factor by an ELISA according to the manufacturer's instructions for the ELISA kit (R\&D Systems, Minneapolis, MN, USA).

Cell cycle analysis. HUVECs were seeded in 60-mm plates as described above. After incubation for $72 \mathrm{~h}$, cells were trypsinized, collected in phosphate-buffered saline (PBS) and fixed on ice followed by $70 \%$ cold ethanol. After treatment with $10 \mu \mathrm{g} / \mathrm{ml}$ RNase, cells were stained with $50 \mu \mathrm{g} / \mathrm{ml}$ propidium iodide (Sigma) for $15 \mathrm{~min}$ at room temperature to prepare for cell cycle analysis. Stained cells were analyzed by flow cytometry (FACSCalibur; BD Biosciences, Franklin Lakes, NJ, USA). The cell cycle information was analyzed using ModFit 3.0 software.

Statistical analysis. All data were subjected to statistical analysis and were reported as the mean \pm standard deviation. ANOVA was used to assess the statistical differences in means among these groups. The criterion for statistical significance was taken as $\mathrm{P}<0.05$ using a two-tailed t-test. The analyses were performed using SPSS 15.0 software.

\section{Results}

Fabrication of micropatterned silk fibroin. In this study, micromolding was used to develop microtopographic structures of silk fibroin. Four defined types of silk fibroin demonstrated features of microtopographical structure and another showed a non-patterned structure as a control (Fig. 1).

Cell behavior on micropatterned silk fibroin surfaces. Fig. 2 shows micrographs of HUVEC imaged at x 160 magnification on silk fibroin with different microtopographic structures or non-patterned structure after $72 \mathrm{~h}$ in culture. Notably, most of the ECs had preferential spreading along the ridges and
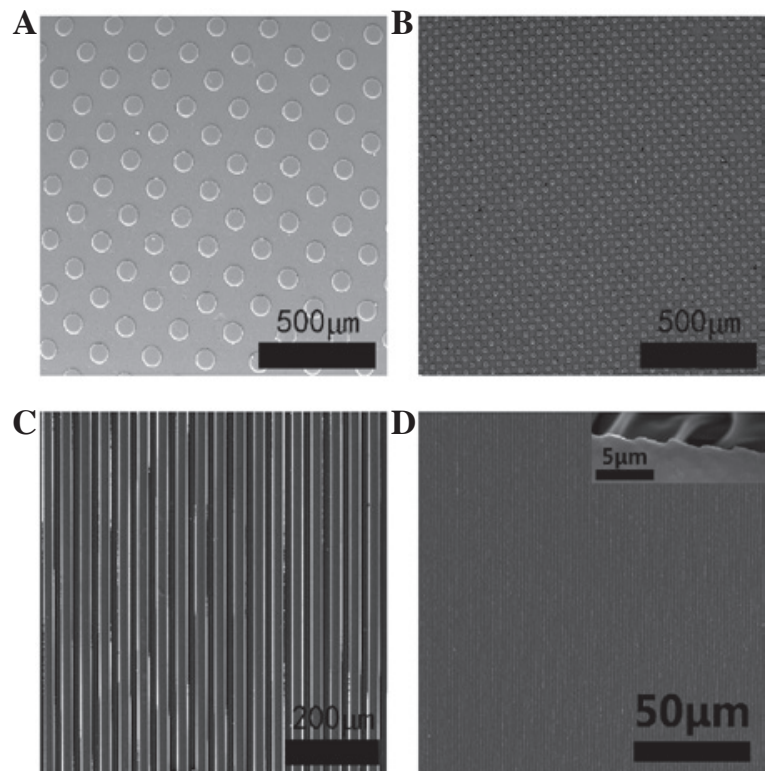

Figure 1. Four samples of silk fibroin showing different features of microtopographical structure under the microscope. In this study, micromolding was used to develop the microtopographic structures of silk fibroin.
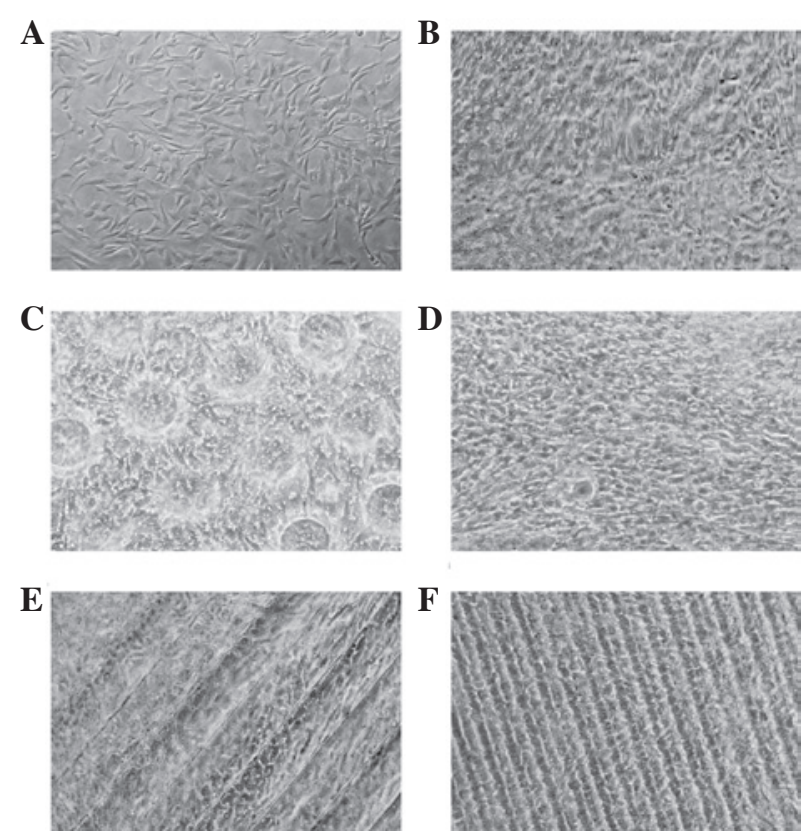

Figure 2. Human umbilical vein endothelial cells (HUVECs) imaged at x160 magnification on silk fibroin with different microtopographic structures or non-patterned structure after $72 \mathrm{~h}$ in culture. (A) Non-silk fibroin. (B) Silk fibroin of non-patterned structure. (C) Silk fibroin of Structure 1. (D) Silk fibroin of Structure 2. (E) Silk fibroin of Structure 3. (F) Silk fibroin of Structure 4.

grooves of the microtopographic structure. The cells clearly show a different adhesion and proliferation behavior depending on the underlying microtopographic structure as described in Table II. Cells in the non-patterned structure group showed a changed morphology, such as fusiform or spherical, compared to the cells of the microtopographic structure groups, which were well attached and stretched, particularly in Structure 1. Meanwhile, the cell debris in the non-patterned structure group was far greater than in the other groups. 
Table II. Micrographs of HUVECs on different types of silk fibroin.

\begin{tabular}{lcc}
\hline Group & Cell debris & Morphology \\
\hline $\begin{array}{l}\text { Non-patterned } \\
\text { structure }\end{array}$ & More & Changed \\
Structure 1 & Less & Very good \\
Structure 2 & Less & Not bad \\
Structure 3 & Less & Good \\
Structure 4 & Less & Good \\
\hline
\end{tabular}

HUVECs, human umbilical vein endothelial cells.

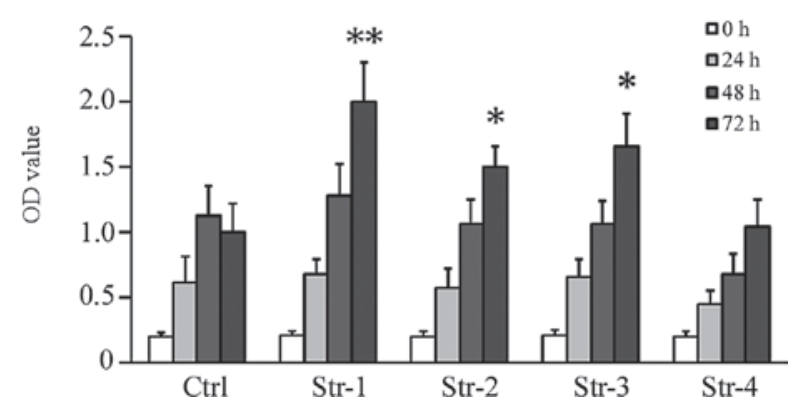

Figure 3. Microtopographic structures had a positive effect on the proliferation of human umbilical vein endothelial cells (HUVECs). Cell proliferation on silk fibroin of 4 types of different microtopographic structures (Str-1, 2, 3 and 4 and a non-patterned structure as a control (Ctrl) were evaluated by Sulforhodamine B (SRB) and detected at a fixed wavelength of $490 \mathrm{~nm}$ after incubation for 24,48 and $72 \mathrm{~h}\left({ }^{* *} \mathrm{P}<0.01,{ }^{*} \mathrm{P}<0.05\right)$.

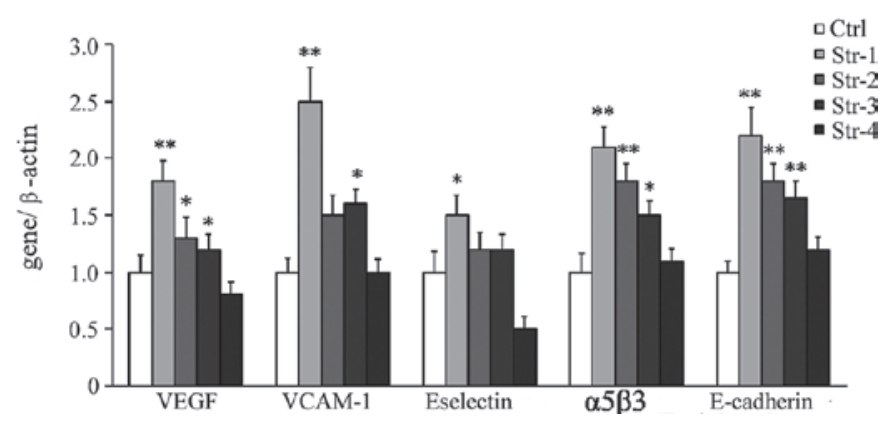

Figure 4. mRNA level of adherent molecules and growth factor of cells growing on silk fibroin of different microstructures were significantly higher than that of the non-patterned structure. Human umbilical vein endothelial cells (HUVECs) were seeded on 4 types of silk fibroin with different microtopographic structures (Str-1, 2, 3 and 4) and one with a non-pattened structure as a control (Ctrl). After incubation for $72 \mathrm{~h}$, mRNA levels of VEGF, VCAM-1, Eselectin, $\alpha 5 \beta 3$ amd E-cadherin were analyzed by real-time PCR $\left({ }^{* * *} \mathrm{P}<0.01,{ }^{*} \mathrm{P}<0.05\right)$.

Effect of microtopographic structure of silk fibroin on cell growth. Cell proliferation evaluated by SRB suggested that cultured HUVECs on silk fibroin with different microtopographic structures or non-patterned structure proliferated from day 1 to 3 of culture and the cell number in Structure 1 increased the most. Compared to the non-patterned structure group, the optical density (OD) values were statistically
$\mathbf{A}$

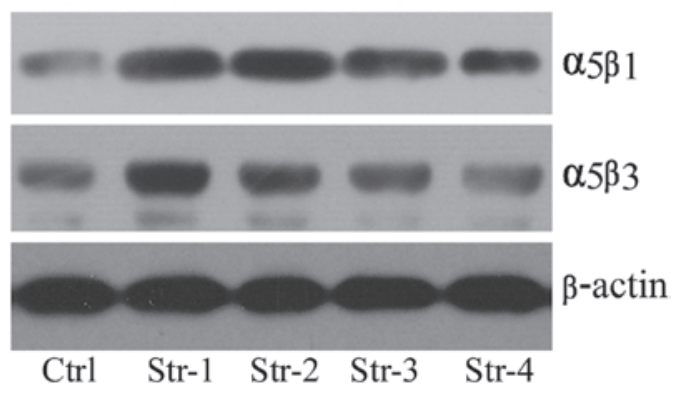

B
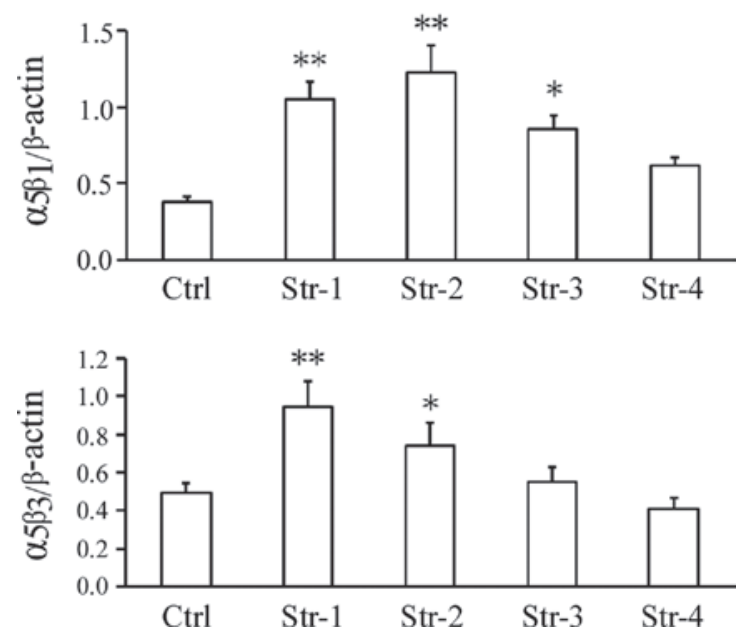

Figure 5. Protein expression of integrin of cells growing on silk fibroin of different microstructures was significantly higher than that of the non-patterned structure. (A) Human umbilical vein endothelial cells (HUVECs) were seeded on 4 types of silk fibroin with different microtopographic structures (Str-1, 2, 3 and 4) and one with a non-pattened structure as a control (Ctrl). After incubation for $72 \mathrm{~h}$, the protein expression levels of $\alpha 5 \beta 1$ and $\alpha 5 \beta 3$ were analyzed by western blotting $\left({ }^{* *} \mathrm{P}<0.01,{ }^{*} \mathrm{P}<0.05\right)$. (B) The protein expression levels of $\alpha 5 \beta 1$ and $\alpha 5 \beta 3$ were calculated by Quantity One software.

significant for the microtopographic structure groups $(\mathrm{P}<0.01$, $\mathrm{P}<0.05)$, with the exception of Structure $4(\mathrm{P}>0.05)$, although for the first 2 days no statistically significant difference was observed, suggesting that HUVECs would proliferate better on silk fibroin with different microtopographic structures (Fig. 3).

Effect of microtopographic structure of silk fibroin on $m R N A$ level and protein expression of growth factor and adhesive molecules. To understand the mechanisms of how most ECs adhere and grow more effectively on silk fibroin with microtopographic structures compared to that of non-patterned structure, we performed molecular studies on the adhesive molecules and growth factor using real-time PCR and western blot analysis. We found that after $72 \mathrm{~h}$, the mRNA level of adherent molecule Eselectin was upregulated in Structure 1, and VCAM-1 was upregulated in Structure 1 and 3; moreover, $\alpha 5 \beta 3$, E-cadherin and VEGF were upregulated in all microtopographic structure groups except Structure 4, compared to the non-patterned structure group, particularly in Structure 1 group $(\mathrm{P}<0.01, \mathrm{P}<0.05$; Fig. 4$)$. In addition, the protein expression of adhesive molecule $\alpha 5 \beta 1$ was upregulated in the microtopographic structure groups except Structure 4, and $\alpha 5 \beta 3$ was upregulated in Structure 1 and 2, compared to the non-patterned structure group $(\mathrm{P}<0.01, \mathrm{P}<0.05$; Fig. 5). 
$\mathbf{A}$

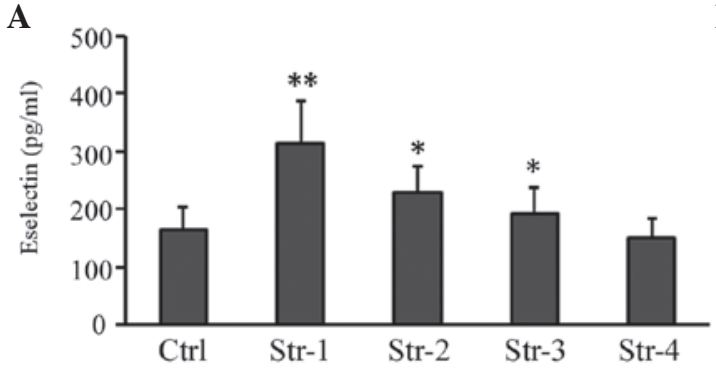

B

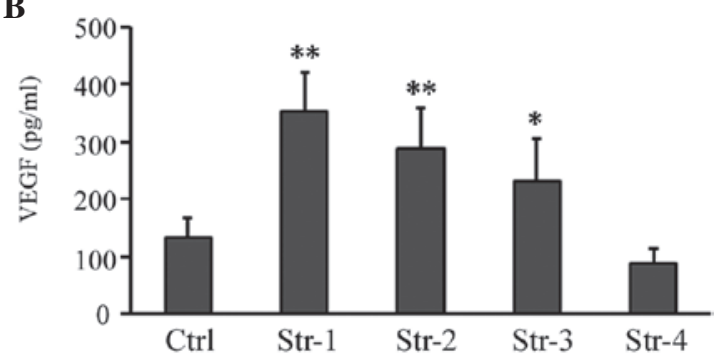

C

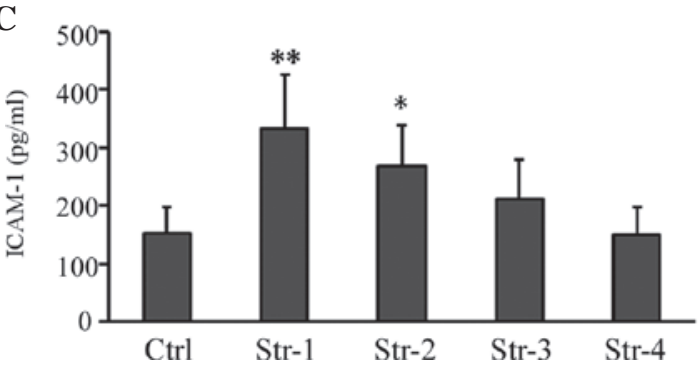

D

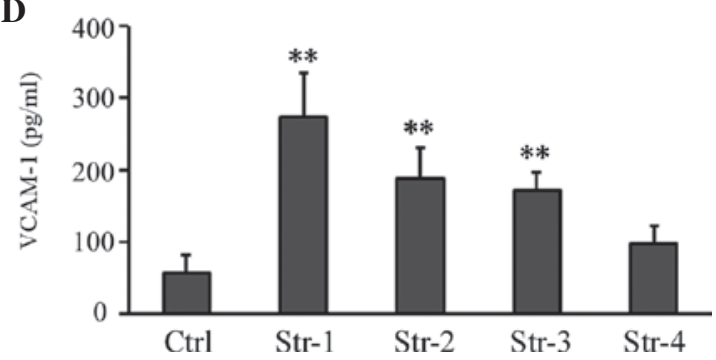

Figure 6. Protein factor secreted in the medium of cells growing on silk fibroin of different microstructures was significantly higher than that of the nonpatterned structure. Human umbilical vein endothelial cells (HUVECs) were seeded on 4 types of silk fibroin with different microtopographic structures (Str-1, 2,3 and 4) and one with a non-pattened structure as a control (Ctrl). After incubation for $72 \mathrm{~h}$, the secretion of growth factor and molecular factors was analyzed by ELISA; (A) Eselectin, (B) VEGF, (C) ICAM-1 and (D) VCAM-1. ${ }^{* *} \mathrm{P}<0.01,{ }^{*} \mathrm{P}<0.05$.

A

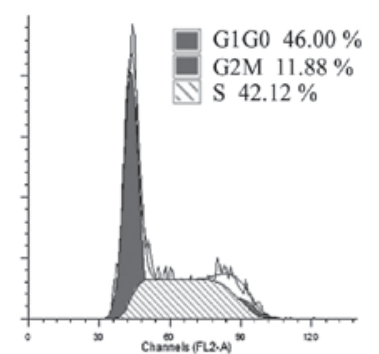

Ctrl

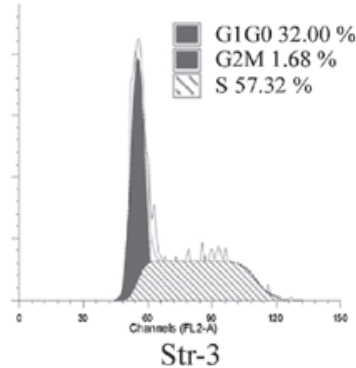

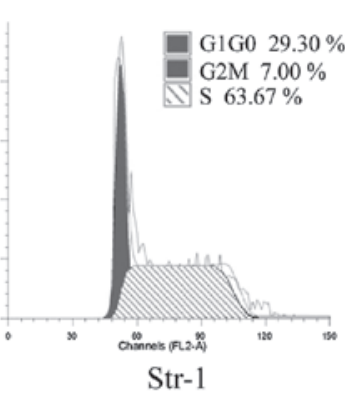

Str-1

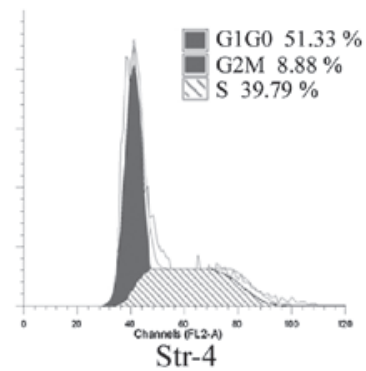

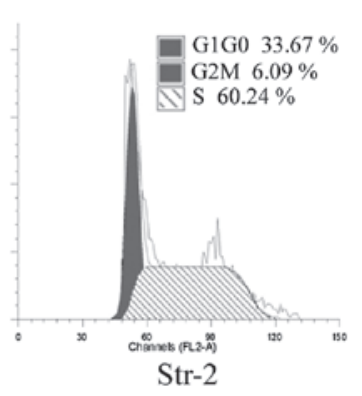

Str-2

$\mathbf{B}$

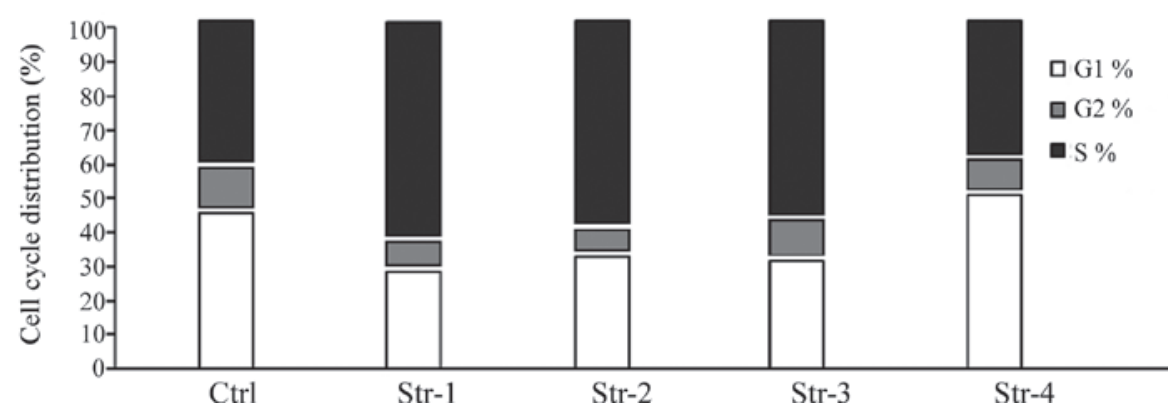

Figure 7. Frequency of cells growing on silk fibroin of different microstructures at the S or G1 phase was markedly increased or decreased compared to that of the non-patterned structure. (A) Human umbilical vein endothelial cells (HUVECs) were seeded on 4 types of silk fibroin with different microtopographic structures (Str-1, 2, 3 and 4) and one with a non-pattened structure as a control (Ctrl). After incubation for $72 \mathrm{~h}$, cells were fixed on ice followed by $70 \%$ cold ethanol and stained with propidium iodide. Stained cells underwent cell cycle analysis by flow cytometry. (B) The cell cycle information was analyzed using ModFit3.0 software. 
Table III. Cell cycle analysis of HUVECs.

\begin{tabular}{lcrc}
\hline Group & $\mathrm{G} 0 / \mathrm{G} 1(\%)$ & $\mathrm{S}(\%)$ & $\mathrm{G} 2 / \mathrm{M}(\%)$ \\
\hline $\begin{array}{l}\text { Non-patterned } \\
\text { structure }\end{array}$ & $46.00 \pm 4.58$ & $11.88 \pm 2.37$ & $42.12 \pm 2.78$ \\
Structure 1 & $29.33 \pm 8.08$ & $7.00 \pm 1.76$ & $63.67 \pm 6.36$ \\
Structure 2 & $33.67 \pm 8.02$ & $6.09 \pm 2.07$ & $60.24 \pm 7.08$ \\
Structure 3 & $32.00 \pm 3.61$ & $10.68 \pm 3.35$ & $57.32 \pm 5.58$ \\
Structure 4 & $51.33 \pm 3.51$ & $8.88 \pm 0.73$ & $39.79 \pm 4.25$ \\
\hline
\end{tabular}

HUVECs, human umbilical vein endothelial cells.

Effect of microtopographic structure of silk fibroin on secretion of protein factor. The influence of microtopographic structure on protein factor secreted in the HUVEC culture supernatant was further investigated using ELISA. We found that after $72 \mathrm{~h}$ in culture, the secretion of growth factor VEGF and adhesive molecules Eselectin and VCAM-1 increased significantly in microtopographic structure groups except Structure 4. Moreover, the secretion of adhesive molecule ICAM-1 increased significantly in Structure 1 and 2 groups (particularly in the former), compared with the non-patterned structure group $(\mathrm{P}<0.01, \mathrm{P}<0.05$; Fig. 6$)$.

Effect of microtopographic structure of silk fibroin on cell cycle. In line with the cell proliferation results, Fig. 7 shows that, with the exception of Structure 4, the frequency of cells at the $\mathrm{S}$ or G1 phase was markedly increased or decreased in the microtopographic structure groups compared to the non-patterned structure group $(\mathrm{P}<0.01, \mathrm{P}<0.05$; Table III).

\section{Discussion}

One of the currently discussed concepts to improve the vascularization of metallic stents is the prevascularization by including vascular ECs. A wide variety of coatings have been explored for stents, targeting the objective to promote endothelialization. The success of such biomaterial is dependent on its ability to support the growth and functioning of the cells growing on it. Prior studies $(12,13)$ showed that silk fibroin had unique properties that fulfill many of the requirements for biomaterial scaffolds and could be used as a coating applied to stent for the culture of human ECs. It was demonstrated that the biophysical environment consisting of microstructures could influence the cellular behavior; however, whether the silk fibroin of different microstructures could support the growth of human ECs and exert effects on endothelial phenotypes or functions have never been discussed.

In this study, silk fibroin of microtopographic structure with four different patterns developed by micromolding were synthesized to evaluate the response of vascular ECs to topographic cues. Laser and UV-based patterning are two prevalent alternative methodologies for engineering and micromolding the surface with specific patterns (18), allowing enhanced resolution despite the higher costs. Of these methods, we adopted the former. We used HUVECs in our experiment since this endothelial cell type is of embryonic origin and of the macrovascular type, whereas ECs involved in inflammation, healing and vascularization are primarily of microvascular origin.

In the present study, we provided quantitative data in order to gain more insight into the effect of the microstructure of silk fibroin on cell growth and further investigated the mechanism of it. The response of HUVECs growing on non-patterned and microtopographic structure silk fibroin scaffolds was compared, with emphasis on cell morphology, proliferation, cell cycle, expression of adhesive molecules and secretion of relevant protein factors.

Our results suggested that the morphology of the HUVECs could be influenced by the microstructure of silk fibroin. It has been reported that cells align along microfeatures in a process resulting from the rearrangement of the cellular cytoskeleton $(18,19)$ and that the cells form cytoplasm extensions and cellular associations over different ridges while populating the groove of the pattern. Cells may have different morphologies on sensing the distinct surface topography and in aggregate, a microtopographic structure would be more helpful to the morphology of the cells than a non-patterned structure.

We also found that silk fibroin of microtopographic structure had positive effects on endothelial cell attachment, with respect to the adhesive molecules expressed intracytoplasm and secreted in the medium, suggesting the potential ability of the cells induced by the microtopographic structure to synthesize, adapt and produce adhesive proteins, to ensure effective adhesion on the surface. In light of the fact that the microstructure influenced the morphology of cells, it is obvious that there is an initial correlation between the attachment and the morphology of cells, which is supported by our observation that cells on silk fibroin of non-patterned structure were round in shape and the substrate demonstrated a low ability to stimulate cell adhesion. However, the mechanism of this requires further investigation.

It is reported that changes in cell shape impacted by the microstructure of the biomaterial have been implicated in alterations in the cell cycle which would directly influence the proliferative state of ECs (20). In our studies, HUVECs demonstrated a significant change in proliferation and cell cycle on the silk fibroin of microstructures compared with that of the non-patterned structure, which was consistent with the morphology of the cells.

Of all the microtopographic structures, the behaviors and inherent phenomena of the cells were particularly influenced by Structure 1, containing arrays of large holes within a flat surface. As most of the cells were inclined to spread along the ridges and grooves of the microtopographic structure, we supposed that an architecture like Structure 1, possessing a great number of large holes, may afford more space for cells to adhere and thus obtain the best advantage. However, the mechanism has to be further investigated.

\section{Acknowledgements}

This study was supported by the Fundamental Research Projects fund (no. 09JC1403200) sponsored by the Shanghai Science and Technology Committee. 


\section{References}

1. Hinohara T: Percutaneous coronary intervention: current perspective. Keio J Med 50: 152-160, 2001.

2. Alfonso F, Pérez-Vizcayno MJ, Cruz A, García J, JimenezQuevedo P, Escaned J and Hernandez R: Treatment of patients with in-stent restenosis. EuroIntervention 5 (Suppl D): D70-D78, 2009.

3. Thansayari P, Kathir K, Celemajer DS and Adams MR: Endothelial dysfunction and restenosis following percutaneous coronary intervention. Int J Cardiol 119: 362-367, 2007.

4. Bonetti PO, Lerman LO and Lerman A: Endothelial dysfunction: a marker of atherosclerotic risk. Arterioscler Thromb Vasc Biol 23: 168-175, 2003.

5. Kipshidze N, Baker J and Nikolaychik N: Fibrin coated stents as an improved vehicle for endothelial cell seeding. Circulation 90: I-597, 1994

6. Flugelman MY, Virmani R, Leon MB, Bowman RL and Dichek DA: Genetically engineered endothelial cells remain adherent and viable after stent deployment and exposure to flow in vitro. Circ Res 70: 348-354, 1992.

7. Scott NA, Candal FJ, Robinson KA and Ades EW: Seeding of intracoronary stents with immortalized human microvascular endothelial cells. Am Heart J 129: 860-866, 1995.

8. Van der Giessen WJ, Serruys PW, Visser WJ, Verdouw PD, van Schalkwijk WP and Jongkind JF: Endothelialization of intravascular stents. J Intervent Cardiol 1: 109-120, 1988.

9. Dichek DA, Neville RF, Zwiebel JA, Freeman SM, Leon MB and Anderson WF: Seeding of intravascular stents with genetically engineered endothelial cells. Circulation 80: 1347-1353, 1989.

10. Chen MC, Liang HF, Chiu YL, Chang Y, Wei HJ and Sung HW: A novel drug-eluting stent spray-coated with multi-layers of collagen and sirolimus. J Control Release 108: 178-189, 2005.

11. Thierry B, Winnik FM, Merhi Y, Silver J and Tabrizian M: Bioactive coatings of endovascular stents based on polyelectrolyte multilayers. Biomacromolecules 4: 1564-1571, 2003.
12. Zhang X, Baughman CB and Kaplan DL: In vitro evaluation of electrospun silk fibroin scaffolds for vascular cell growth. Biomaterials 29: 2217-2227, 2008.

13. Wang X, Zhang X, Castellot J, Herman I, Iafrati M and Kaplan DL: Controlled release from multilayer silk biomaterial coatings to modulate vascular cell responses. Biomaterials 29: 894-903, 2008

14. Altman GH, Diaz F, Jakuba C, Calabro T, Horan RL, Chen J, Lu H, Richmond J and Kaplan DL: Silk-based biomaterials. Biomaterials 24: 401-416, 2003.

15. Diener A, Nebe B, Lüthen F, Becker P, Beck U, Neumann HG and Rychly J: Control of focal adhesion dynamics by material surface characteristics. Biomaterials 26: 383-392, 2005.

16. Karuri NW, Porri TJ, Albrecht RM, Murphy CJ and Nealey PF: Nano- and microscale holes modulate cell-substrate adhesion, cytoskeletal organization, and -betal integrin localization in SV40 human corneal epithelial cells. IEEE Trans Nanobioscience 5: 273-280, 2006.

17. Jaffe EA, Nachman RL, Becker CG and Minick CR: Culture of human endothelial cells derived from umbilical veins. Identification by morphologic and immunologic criteria. J Clin Invest 52: 2745-2756, 1973.

18. Teixeira AI, Nealey PF and Murphy CJ: Responses of human keratocytes to micro- and nano structured substrates. J Biomed Mater Res A 71: 369-376, 2004.

19. Rebollar E, Frischauf I, Olbrich M, Peterbauer T, Hering S, Preiner J, Hinterdorfer P, Romanin C and Heitz J: Proliferation of aligned mammalian cells on laser-nanostructured polystyrene. Biomaterials 29: 1796-1806, 2008.

20. Roca-Cusachs P, Alcaraz J, Sunyer R, Samitier J, Farré R and Navajas D: Micropatterning of single endothelial cell shape reveals a tight coupling between nuclear volume in G1 and proliferation. Biophys J 94: 4984-4995, 2008. 